\title{
Peak to Average Power Reduction Using Amplitude and Sign Adjustment
}

\author{
Masoud Sharif, Cedric Florens, Maryam Fazel, and Babak Hassibi \\ Department of Electrical Engineering \\ California Institute of Technology \\ Pasadena, CA 91125
}

\begin{abstract}
In this paper, we propose a method to reduce the peak to mean envelope power ratio (PMEPR) of multicarrier signals by modifying the constellation. For MPSK constellations, we minimize the maximum of the multicarrier signal over the sign and amplitude of each subcarrier. In order to find an efficient solution to the aforementioned non-convex optimization problem, we present a suboptimal solution by first optimizing over the signs using the result of [1], and then optimizing over the amplitudes given the signs. We prove that the minimization of the maximum of a multicarrier signal over the amplitude of each subcarrier can be written as a convex optimization problem with linear matrix inequality constraints. We also generalize the idea to other constellations such as 16QAM. Simulation results show that by an average power increase of $0.21 \mathrm{db}$ and not sending information over the sign of each subcarrier, PMEPR can be decreased by 5.1 db for a system with 128 subcarriers.
\end{abstract}

\section{INTRODUCTION}

High peak to mean envelope power ratio (PMEPR) of multicarrier signal is one of the major obstacles in implementing OFDM, xDSL, and other broadband multicarrier systems. The occurrence of the large peaks in the signal seriously hampers the efficiency of the power amplifier.

Over the years, different schemes have been proposed for PMEPR reduction such as coding, deliberate clipping, selective mapping (SLM), reserved carriers, and tone injection [2], [3], [4], [5], [6], [7]. In all these schemes, there is always a trade off between PMEPR and other parameters in the systems, including coding rate, average power, signal distortion, and bandwidth. Methods like coding usually give a worst case guarantee on the PMEPR, on the other hand, there are other methods such as SLM that improve the probability distribution of PMEPR, i.e. reduce the probability of encountering large PMEPR.

Recently, in [1], an algorithm has been proposed to choose the sign of each subcarrier in order to reduce the PMEPR. In this paper, we further generalize this idea and adjust the sign and amplitude of each subcarrier. The price to adjust the amplitude of the subcarrier is a slight increase in the average power. Even though the optimization over the signs is not a convex optimization problem, we show that the amplitude

This work was supported in part by the National Science Foundation under grant no. CCR-0133818, by the office of Naval Research under grant no. N00014-02-1-0578, and by Caltech's Lee Center for Advanced Networking. optimization can be written as a convex optimization problem using the Bounded Real Lemma [8]. This enables us to efficiently solve the problem and add more practical constraints to the problem like limiting the amplitude of each subcarrier in order to bound the peak to average in frequency domain.

Our approach can be considered as a method to refine the constellation for PMEPR reductions. Other methods to shape the constellation have appeared in [9] and [7] to reduce the maximum of the samples of the multicarrier signal. In [7], extending the number of constellation points is proposed, however, in [9] outer points in the constellation are allowed to move within margin-preserving constraints. In this paper, we consider a different constellation modification and we further show that reducing the peak of the continuous multicarrier signal by optimizing the amplitude of the subcarriers is a convex optimization problem. In our approach we first reduce the peak by optimizing over the signs of the multicarrier signal which is not a convex problem.

Simulation results show that the PMEPR can be significantly reduced by using just $0.21 \mathrm{db}$ (i.e. $5 \%$ ) average power increase. More specifically, for a system with 128 subcarriers, and considering the peaks with probability less than $10^{-2}$ as negligible, PMEPR is reduced from 10.3 to 3.1 , i.e. $5.1 \mathrm{db}$ PMEPR improvement.

The paper is organized as follows: Section 2 introduces our notations and the statement of the problem, and furthermore reviews the sign optimization algorithm. Section 3 deals with amplitude optimization and proves that it is a convex problem using bounded real lemma. Simulations results are presented in Section 4 and Section 5 concludes the paper.

\section{Definitions And Problem Statement}

In this paper, we consider a normalized multicarrier signal $s_{C}(\theta)$ that consists of $n$ subcarriers. More specifically,

$$
s_{C}(\theta)=\sum_{i=1}^{n} c_{i} e^{j \theta i}
$$

where $C=\left(c_{1}, \ldots, c_{n}\right)$ is the modulating vector, $c_{i}$ 's are chosen from some constellations like MPSK or 16QAM, and $\theta$ denotes time. Clearly, if $c_{i}$ 's are chosen from BPSK constellation and they add up coherently, $s_{C}(\theta)$ will have a large peak of order $n$. Therefore it is of great practical interest 


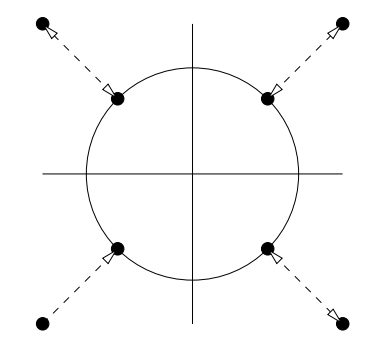

Fig. 1. Modified QPSK constellation

to reduce the peak of $s_{C}(\theta)$ without excessively increasing the average power and introducing large peaks in the modulating codeword $C$.

As a measure for the fluctuation of the multicarrier signal, we may define the PMEPR of $C$ as

$$
\operatorname{PMEPR}(C)=\max _{0 \leq \theta \leq 2 \pi} \frac{\left|s_{C}(\theta)\right|^{2}}{E\left\{\sum_{i=1}^{n}\left|c_{i}\right|^{2}\right\}} .
$$

where the denominator is the average power of $s_{C}(\theta)$. Thus, if $c_{i}$ 's are chosen independently from a constellation with average power of $P_{a v}$, then $E\left\{\sum_{i=1}^{n}\left|c_{i}\right|^{2}\right\}=n P_{a v}$.

In this paper, we consider the PMEPR reduction by adjusting the sign and amplitude of each subcarrier. This method is a more general version of the scheme that has been recently proposed in [1] and it is shown that by just adjusting the signs we can achieve constant PMEPR with a little rate hit for large $n$ [10]. Here we first consider MPSK constellations and we then generalize the idea to other constellations as well.

Here is the statement of the problem: For any given complex vector $C=\left(c_{1}, \ldots, c_{n}\right)$ where $c_{i}$ 's are chosen from any MPSK constellation, find the solution to the following optimization problem:

$$
\begin{array}{ll}
\underset{\epsilon, u}{\operatorname{minimize}} & \max _{0 \leq \theta \leq 2 \pi}\left|\sum_{i=1}^{n} \epsilon_{i}\left(1+u_{i}\right) c_{i} e^{j \theta i}\right| \\
\text { subject to } & \epsilon_{i} \in\{+1,-1\} \\
& 0 \leq u_{i} \leq u_{\max } \\
& \sum_{i=1}^{n}\left|c_{i}\right|^{2}\left(1+u_{i}\right)^{2} \leq(1+\eta) n P_{a v}
\end{array}
$$

where $\epsilon=\left(\epsilon_{1}, \ldots, \epsilon_{n}\right)$ and $u \in \mathbf{R}^{n}$ are the optimization variables, $u_{i}$ denotes the amplitude variations of the $i$ 'th subcarrier, and $\eta$ denotes the average power increase. In order to limit the variation of the new modulating vector $\left(\epsilon_{1}\left(1+u_{1}\right) c_{1}, \ldots, \epsilon_{n}\left(1+u_{n}\right) c_{n}\right)$, we further constrain $u_{i}$ to be less than $u_{\max }$. The last constraint also implies that the average power increase is controlled by the parameter $\eta$. Using our scheme, the receiver is not required to know the vectors $\epsilon$ and $u$ for decoding.

In summary, the price for reducing the PMEPR with our scheme is a slight increase in the average power, $\eta$, and sending no information over the sign of each subcarrier. For instance, Fig. 1 and 2 show the modified QPSK and 16QAM constellations, respectively.

It is worth noting that with this variation of the constellation,

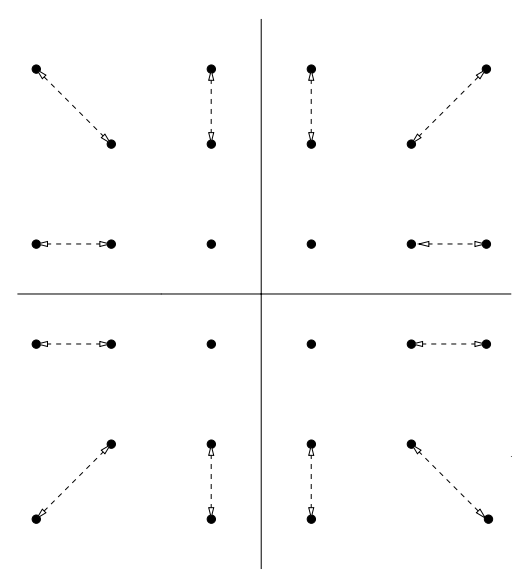

Fig. 2. Modified 16QAM constellation

the minimum distance of the constellation points remains unchanged. For constellations other than MPSK, we let outer points in the constellation move such that the minimum distance of the constellation points does not change. This is shown for the 16QAM constellation in Fig. 2.

Clearly the optimization problem as stated in (3) is not convex due to having an integer constraint, i.e. $\epsilon_{i} \in\{+1,-1\}$. However assuming that $u_{i}=0$ for all $i$, we can find a suboptimal solution for the signs using the result of [1]. Afterwards, given $c_{i}$ 's and $\epsilon_{i}$ 's, we show in Section 4 that the optimization over $u_{i}$ 's is convex and can be done very efficiently.

In what follows, we briefly review the sign optimization algorithm. In [1], an algorithm is proposed to design the signs and it is proved that the PMEPR of the resulting codeword defined in (2) is less than $\alpha \log n$ for any $n$ where $\alpha$ is a constant independent of $n$. Here is the algorithm:

Algorithm 1. For any $C=\left(c_{1}, \ldots, c_{n}\right)$, let $k$ be an integer

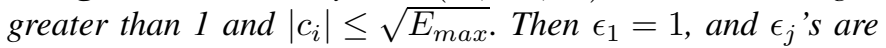
recursively determined as the minus sign of

$$
\sum_{p=1}^{2 k n} \sinh \left\{\alpha^{*} \sum_{r=1}^{j-1} \epsilon_{r} a_{p r}\right\} \sinh \left(\alpha^{*} a_{p j}\right) \prod_{r=j+1}^{n} \cosh \left\{\alpha^{*} a_{p r}\right\} .
$$

for $j=2, \ldots, n$, where $\alpha^{*}=\sqrt{\frac{2 \log 4 k n}{n E_{\max }}}$,

$$
a_{p i}= \begin{cases}\operatorname{Re}\left\{c_{i} e^{j \theta_{p} i}\right\} & 1 \leq p \leq k n, \\ \operatorname{Im}\left\{c_{i} e^{j \theta_{p} i}\right\} & k n+1 \leq p \leq 2 k n,\end{cases}
$$

and $\theta_{p}=\frac{2 \pi p}{k n}$.

It can be shown that the PMEPR of the codeword $C_{\epsilon}=$ $\left(\epsilon_{1} c_{1}, \ldots, \epsilon_{n} c_{n}\right)$ is less than $\gamma \log n$ for any $n$ where $\gamma$ is a constant independent of $n$. Even though, Algorithm 1 does not give us the best signs, it is shown in [1] that it can significantly reduce the PMEPR. Now by further optimizing over the $u_{i}$, we can further reduce the PMEPR at the price of an slight increase in the average power. This gives us another degree of freedom to trade the PMEPR with negligible average power 
increase and without deteriorating the minimum distance of the constellation.

It is also worth noting that one might ask whether changing the order of the optimization might improve the PMEPR reduction. Intuitively, balancing the maximum of a multicarrier signal which is already fairly balanced by optimizing over the sign of each subcarrier, requires less average power increase $\eta$ than the case where we first optimize the constellation over $u_{i}$ 's. Simulation results also confirm this.

\section{Amplitude Adjustment Using Convex OPTIMIZATION}

In this section, we solve the problem of minimizing the peak of the multicarrier signal over $u_{i}$ 's given the signs and the information symbols $c_{i}$ 's and we show that it is a convex problem with a Linear Matrix Inequality (LMI) constraint [11]. We further present a relaxation of the problem that leads to an approximate solution with less complexity by minimizing the maximum of the samples of the multicarrier signal.

First of all we notice the fact that

$$
\sum_{i=1}^{n} c_{i} \epsilon_{i}\left(1+u_{i}\right) z^{-i}=H(z I-F)^{-1} G,
$$

where $H^{t}=\left[c_{1} \epsilon_{1}\left(1+u_{1}\right), \ldots, c_{n} \epsilon_{n}\left(1+u_{n}\right)\right], z=e^{j \theta}, G^{t}=$ $[1,0, \ldots, 0]$, and

$$
F=\left[\begin{array}{cccc}
0 & \ldots & 0 & \\
1 & 0 & \ldots & \\
\vdots & \vdots & & \\
0 & \ldots & 1 & 0
\end{array}\right]
$$

Given the $\epsilon_{i}$ 's and $c_{i}$ 's, we can then restate (3) as the following optimization problem,

$$
\begin{array}{ll}
\operatorname{minimize} & \gamma \\
\text { subject to } & \left\|H(z I-F)^{-1} G\right\|_{\infty} \leq \gamma \\
& 0 \leq u_{i} \leq u_{\max } \\
& \sum_{i=1}^{n}\left|c_{i}\right|^{2}\left(1+u_{i}\right)^{2} \leq(1+\eta) n P_{a v} .
\end{array}
$$

In order to show that the above problem is convex, we use the Bounded Real Lemma [8]:

Lemma 1: (Bounded Real Lemma) Suppose $\gamma>0$ and $F$ is stable. Then the following two statements are equivalent:

(i)

$$
\left\|H(z I-F)^{-1} G\right\|_{\infty}<\gamma .
$$

(ii) There exists a Hermitian $Y$ such that

$$
\mathcal{H}=\left[\begin{array}{ccc}
-Y+F^{*} Y F & H^{*} & F^{*} Y G \\
H & \gamma I & 0 \\
G^{*} Y F & 0 & \gamma I+G^{*} Y G
\end{array}\right]>0 .
$$

Since the matrix $\mathcal{H}$ is linear in the entries of the matrix $H$, we can see that the constraint in the optimization problem of (6) is a Linear Matrix Inequality (LMI) [11].

Therefore, given the values of $\epsilon_{i}$ 's and $c_{i}$ 's, we can state the minimization in (3) over $u_{i}$ 's as the following convex optimization problem:

$$
\begin{array}{ll}
\operatorname{minimize} & \gamma \\
\text { subject to } & \mathcal{H} \geq 0 \\
& 0 \leq u_{i} \leq u_{\max } \\
& \sum_{i=1}^{n}\left|c_{i}\right|^{2}\left(1+u_{i}\right)^{2} \leq(1+\eta) n P_{a v} .
\end{array}
$$

where $H^{t}=\left[c_{1} \epsilon_{1}\left(1+u_{1}\right), \ldots, c_{n} \epsilon_{n}\left(1+u_{n}\right)\right]$, and the matrix $Y$ in $\mathcal{H}$ is Hermitian.

This problem is a Semi-Definite program (SDP) and can be solved globally and efficiently using interior-point methods. Software packages exist that implement these methods; we use the recent package SeDuMi 1.02 [12].

Since the size of the LMI in the above SDP is relatively large, the computational load is still high for practical purposes. In fact the complexity is $O\left(n^{6}\right)$ even though exploiting the structure of the LMI can lead to faster implementations. Another way to lower the computational load with very little loss in accuracy is to discretize $s_{C}(\theta)$ and then solve the discretized problem. That is, instead of minimizing the maximum of $s_{C}(\theta)$ over $1 \leq \theta \leq 2 \pi$, we consider minimization of the maximum of $k n$ uniform samples of $s_{C}(\theta)$ at $\theta_{p}=\frac{2 \pi p}{k n}$ for $p=1, \ldots, k n$. This certainly has much less complexity and can be written as a quadratically-constrained quadratic program [11], which is solved much more efficiently than the original SDP. We use SeDuMi for solving this problem as well. Furthermore, using the relationship between the maximum of $s_{C}(\theta)$ over $\theta$ and the maximum over $\theta_{i}$, we can make our approximation practically accurate by choosing $k=4$ [13], [14].

More specifically, this optimization problem can be written as,

$$
\begin{array}{ll}
\operatorname{minimize} & \gamma \\
\text { subject to } & 0 \leq u_{i} \leq u_{\max } \\
& \left|\operatorname{Re}\left\{s_{C}\left(\theta_{p}\right)\right\}\right|^{2}+\left|\operatorname{Im}\left\{s_{C}\left(\theta_{p}\right)\right\}\right|^{2} \leq \gamma \\
& \text { for } p=1, \ldots, k n \\
& \sum_{i=1}^{n}\left|c_{i}\right|^{2}\left(1+u_{i}\right)^{2} \leq(1+\eta) n P_{a v} .
\end{array}
$$

Simulation result show that the result of (7) and (8) is very close by choosing $k=4$. Therefore, in the simulations part we solve the problem in (8) to optimize over $u_{i}$ 's instead of solving (7) which requires more computation.

\section{Simulation Results}

As we discussed in the previous sections, there is a trade off between PMEPR reduction and average power increase, $\eta$, and also the range of variation for $u_{i}$ 's, i.e. $u_{\max }$. In this section we carry out simulations to explore this trade off for $n=$ 64 and $n=128$ and for QPSK and 16QAM constellations. The algorithm for designing the signs is applicable to any symmetric constellation. For the amplitude variation of the constellation points, we use the schemes shown in Fig. 1 and Fig. 2, for QPSK and 16QAM respectively.

Fig. 3 shows the CCDF (complementary cumulative distribution function) of PMEPR when $c_{i}$ 's are chosen from QPSK constellation and for different average power increases. Clearly, we need at least $0.21 \mathrm{db}(\eta=0.05)$ average power 


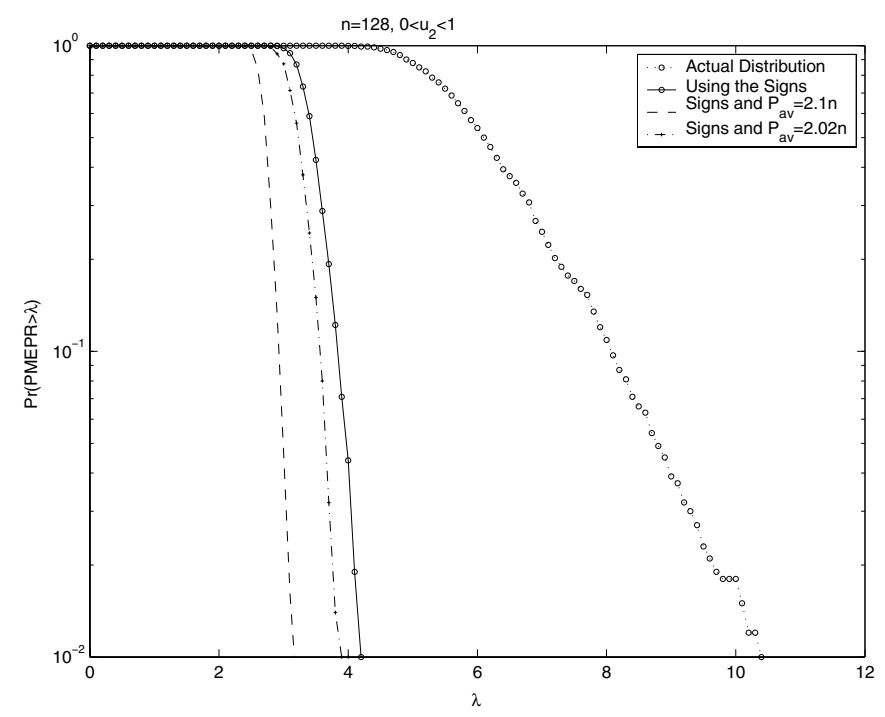

Fig. 3. CCDF of the PMEPR for QPSK by optimizing over the $\epsilon_{i}$ 's and $u_{i}$ 's for $n=128, u_{\max }=1$, and $\eta=0.01,0.05$.

increase, to get a noticeable PMEPR reduction after optimizing over the signs. As Fig. 3 suggests for $n=128$, the PMEPR can be decreased from 10.5 to 4.5 with just using the signs and this can be further pushed down to 3.1 by also optimizing over the $u_{i}$ 's with a little average power increase.

We can further do the simulations for a wider range of $u_{i}$, i.e $u_{\max }=2$. As Fig. 4 shows for $n=128$, we can further reduce the PMEPR by allowing more degree of freedom to each point, however, this causes large peak to average power ratios for the $c_{i}$ 's which is not practically favorable.

Fig. 5 also shows the PMEPR reduction when $c_{i}$ 's are chosen from 16QAM constellation and the variation of the constellation points is as in Fig. 2. In summary, simulation results suggest that by expanding the constellation and increasing the average power by $0.21 \mathrm{db}$, the PMEPR of multicarrier signals can be decreased dramatically, i.e. from 10.5 to 3.1 for $n=128$.

\section{CONCLUSION}

We presented a method to modify the constellation in order to reduce the PMEPR. This is done by minimizing the peak value of the multicarrier signal over the signs and amplitudes of the subcarriers with a slight increase in the average power. Since the problem is not a convex problem, we first used the algorithm in [1] to find suboptimal solution for the signs and then we used the convex optimization algorithm to optimize over the signs. Simulation results show significant improvement on PMEPR.

\section{REFERENCES}

[1] M. Sharif and B. Hassibi, "A deterministic algorithm that achieves PMEPR of $c \log n$ for multicarrier signals," Proc. IEEE ICASSP, pp. 540-543, 2003.

[2] K .G. Paterson and V. Tarokh, "On the existence and construction of good codes with low peak to average power ratios," IEEE Trans. Inform., vol. 46, no. 6, pp. 1974-1986, Sep. 2000.

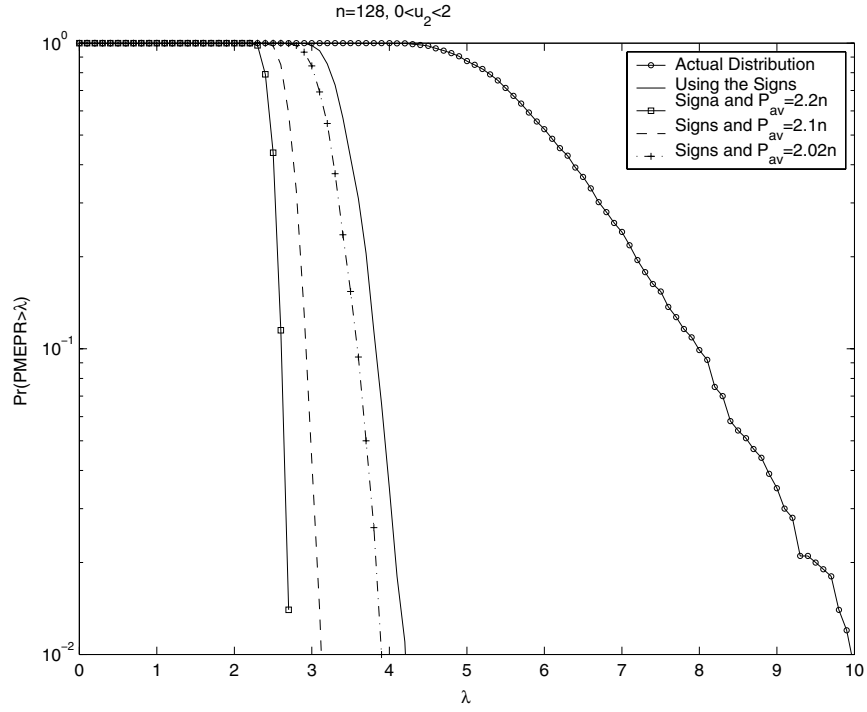

Fig. 4. CCDF of the PMEPR for QPSK by optimizing over the $\epsilon_{i}$ 's and $u_{i}$ 's for $n=128, u_{\max }=2$, and $\eta=0.01,0.05,0.1$.

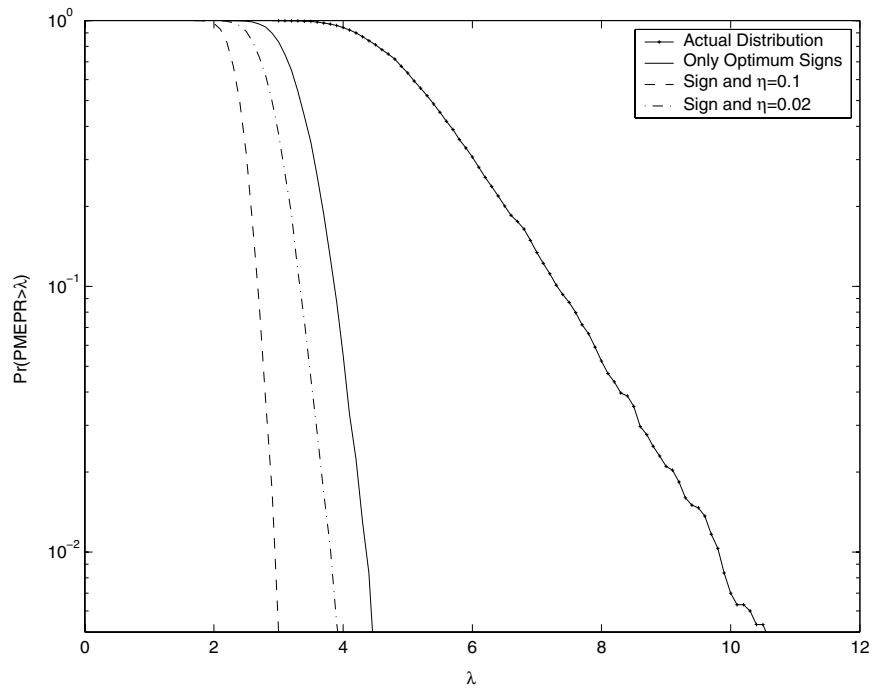

Fig. 5. CCDF of the PMEPR for 16QAM constellation by optimizing over the $\epsilon_{i}$ 's and $u_{i}$ 's for $n=64, \eta=0.02,0.1$, and the constellation modification is according to Fig. 2 with $u_{\max }=0.3$ for outer points.

[3] K .G. Paterson, "Generalized Reed-Muller codes and power control in OFDM modulation," IEEE Trans. Inform., vol. 46, no. 1, pp. 104-120, Jan. 2000.

[4] H. Ochiai and H. Imai, "Performance of the deliberate clipping with adaptive symbol selection for strictly band-limited OFDM systems," IEEE Jour. Selec. Areas Commu., vol. 18, no. 11, pp. 2270-2277, Nov. 2000.

[5] X. Li and L. J. Cimini, "Effects of clipping and filtering on the performance of OFDM," in Proc. IEEE Veh. Tech. Conf., May 1997, pp. $1634-1638$.

[6] S. H. Muller and J. B. Huber, "A comparison of peak power reduction schemes for OFDM," in Proc. IEEE Glob. Comm. Conf., 1997, pp. 1-5.

[7] J. Tellado, Multicarrier modulation with low PAR: applications to DSL and Wireless, Kluwer Academic Publishers, 2000.

[8] B. Hassibi, A. H. Sayed, and T. Kailath, Indefinite-Quadratic estimation 
and control: a unified approach to $H^{2}$ and $H^{\infty}$ theories, SIAM studies in applied and numerical mathematics, 1999.

[9] D. Jones, "Peak power reduction in OFDM and DMT via active channel modification," in Proc. of 32nd Asilomar Conf., Nov. 1999, pp. 10761079.

[10] M. Sharif and B. Hassibi, "On the existence of codes with constant peak to mean envelope power ratio," Proc. IEEE Int. Symp. Info. Theo., 2003.

[11] S. Boyd and L. Vanderberghe, Convex Optimization, to be published, 2003.

[12] J. F. Sturm, "Using sedumi 1.02, a matlab toolbox for optimization over symmetric cones," Optimization Methods and Software, pp. 625-653, 1999.

[13] M. Sharif, M. Gharavi-Alkhansari, and B. H. Khalaj, "On the peak to average power of OFDM signals based on oversampling," IEEE Trans. Comm., vol. 51, no. 1, pp. 72-78, Jan. 2003.

[14] G. Wunder and H. Boche, "Peak value estimation of bandlimited signals from their samples, noise enhancement, and a local characterization in the neighborhood of an extermum," IEEE Trans. Signal Processing, vol. 51, no. 3, pp. 771-780, March 2003. 